\title{
Options for emission allowance allocation under the Eu Emissions Trading Directive
}

\section{Markus Ahman • Lars Zetterberg}

This erratum is being published as a result of Author's name was misspelled. Markus Alhman should be spelled as Markus Ahman. 